\title{
OPTIMAL PLACEMENT OF PIEZOELECTRIC ELEMENT ON A PLATE INTERACTING WITH A FLUID LAYER
}

\author{
Dmitrii A. Oshmarin, Sergey V. Lekomtsev and Natalya V. Sevodina \\ Department of complex problems in mechanics of deformable solids, \\ Institute of Continuous Media Mechanics of the Ural Branch of Russian Academy of \\ Sciences, Perm, Russian Federation.
}

\begin{abstract}
In this paper, we determine the optimal location of the piezoelectric element on the upper surface of a plate, interacting with a layer of a quiescent fluid of finite size. As a quantity, allowing us to evaluate the operating efficiency of a piezoelectric element in damping a single specified mode of structure vibrations, we take the electromechanical coupling coefficient. It is calculated based on the values of the natural frequencies of the system, obtained for the two characteristic performance modes of an electric circuit (open and short circuit modes). The behavior of the piezoelectric element is described by the equations of electrodynamics of deformable electroelastic media in the framework of the quasi-static approximation. The motion of an ideal fluid in the case of small perturbations is considered in the framework of the acoustic approximation. Small strains in a thin plate are determined using the Reissner-Mindlin theory. The numerical implementation of the problem is carried out using the finite element method. The obtained results made it possible to identify situations, where the optimal location of the piezoelectric element can be determined without considering the effect of the fluid.
\end{abstract}

Key words: natural vibrations, ideal fluid, optimization and finite element method.

Cite this Article: Dmitrii A. Oshmarin, Sergey V. Lekomtsev and Natalya V. Sevodina, Optimal Placement of Piezoelectric Element on a Plate Interacting with a Fluid Layer, International Journal of Advanced Research in Engineering and Technology, 10(2), 2019, pp 659-668.

http://iaeme.com/Home/issue/IJARET?Volume=10\&Issue $=2$

\section{INTRODUCTION}

In the last few decades, piezoelectric materials have found wide use in many practical applications in different branches of science and technology. One of the variants of advanced engineering applications is their use in controlling the dynamic characteristics of structures, in particular, vibration damping. When connected to external passive electric circuits, piezoelectric elements attached to the surface of structures comprise devices, which serve to dissipate energy. The piezoelectric effect consists in the transformation of part of the 
mechanical energy of vibrations into the electrical energy, which can dissipate through a shunting circuit as heat or electromagnetic radiation, enabling in such a way the mechanism of passive control of vibrations.

The first studies, which consider the possibility of controlling the dynamic characteristics of structures by attaching an additional electrical impedance to the piezoelement, dated back to the late $70 \mathrm{~s}$ of the last century [1]. However, the first work where this approach was investigated in detail is article [2]. It described the main features of the behavior of structures with shunted piezoelements, including the influence of the external circuit on the mechanical characteristics of the structure. In addition, work [2] introduced an approach for the selection of parameters of external circuits, which was based on the analysis of the transfer function of the system.

In designing modern high-tech structures, which incorporate elements made of piezoelectric materials, one of the most important problems, irrespective of the targeted applications of piezoelectric elements (vibration control [3], sensors and actuators [4], energy harvesting [5], and so on) is the problem of determining their optimal location. To date, although there are hundreds of works devoted to its solution [6], the search for the optimal location of the piezoelectric element in the structure is still the problem of current concern.

In the context of the problems of vibration damping with the help of passive electric circuits connected to a piezoelement, the determination of the optimal location for a piezoelectric element reduces in most cases to a search for a point on the surface of the structure, at which one of the three parameters takes a maximum value. Among these are the deformation energy [6-8], potential difference on the electrode-covered surface of the piezoelectric element $[9,10]$ or the electromechanical coupling coefficient [11-13]. Analysis of the literature [6-13] shows that for numerical simulation, it is most convenient to use an approach based on the electromechanical coupling coefficient. This is explained by the fact that this coefficient is calculated using the values of natural frequencies of structure vibrations obtained for two characteristic modes (open and short circuit modes) [2].

In this paper, the optimal coordinates of the placement of a piezoelectric element on the surface of a plate interacting with a layer of quiescent fluid are determined from the condition for a maximum value of the electromechanical coupling coefficient obtained under the specified modes of vibration. The effect of such hydroelastic interaction on the performance of the piezoelectric element and its optimal location for different types of plate fastening is estimated based on the results of numerical solution of the problem by the finite element method.

\section{MATHEMATICAL MODEL}

The variational equation of motion of the body of volume $V=V_{s}+V_{p}$, consisting of an elastic plate of volume $V_{s}$ and an attached piezoelectric element of volume $V_{p}$, is formulated on the basis of the relations of Reissner - Mindlin theory, linear theory of elasticity and quasi-static Maxwell equations [14-17]. The electrodes applied to the top $\left(S_{t o p}\right)$ and bottom $\left(S_{b o t}\right)$ parts of the surface of piezoelectric body are assumed to be ideal conductors with negligible mass. The final resolving equation in the absence of body forces and free surface charges can be written in the matrix form as

$$
\begin{gathered}
\int_{V_{p}}\left(\left\{\delta \boldsymbol{\varepsilon}_{p}\right\}^{\mathrm{T}}\left[\mathbf{D}_{p}\right]\left\{\boldsymbol{\varepsilon}_{p}\right\}-\left\{\delta \boldsymbol{\varepsilon}_{p}\right\}^{\mathrm{T}}[\mathbf{e}]\{\mathbf{E}\}-\{\delta \mathbf{E}\}^{\mathrm{T}}[\mathbf{e}]^{\mathrm{T}}\left\{\boldsymbol{\varepsilon}_{p}\right\}-\{\delta \mathbf{E}\}^{\mathrm{T}}[\boldsymbol{\beta}]^{\mathrm{T}}\{\mathbf{E}\}\right) d V+ \\
\int_{V_{p}}\left\{\delta \mathbf{u}_{p}\right\}^{\mathrm{T}} \rho_{p}\left\{\ddot{\mathbf{u}}_{p}\right\} d V+\int_{S_{s}}\left\{\delta \boldsymbol{\varepsilon}_{s}\right\}^{\mathrm{T}}\left[\mathbf{D}_{s}\right]\left\{\boldsymbol{\varepsilon}_{s}\right\} d S+\int_{V_{s}}\left\{\delta \mathbf{u}_{s}\right\}^{\mathrm{T}} \rho_{s}\left\{\ddot{\mathbf{u}}_{s}\right\} d V-\int_{S_{\sigma}}\left\{\delta \mathbf{u}_{s}\right\}^{\mathrm{T}}\left\{\mathbf{t}_{s}\right\} d S=0 .
\end{gathered}
$$


Here, the subscripts " $p$ " and " $s$ " refer to the corresponding variables of the piezoelectric element and the plate. The following notations are accepted: column vectors $\{\boldsymbol{\varepsilon}\}$ contain the components of strain tensors and are defined according to the well-known relations $[15,16]$; $[\mathbf{D}]$ are the elastic constant matrices; $[\mathbf{e}],[\boldsymbol{\beta}]$ are the matrices of piezoelectric and dielectric coefficients; $\{\mathbf{E}\}$ is the electric field intensity vector; the potentiality condition is fulfilled for an electric field $-\{\mathbf{E}\}=\left\{\varphi_{, x}, \varphi_{, y}, \varphi_{, z}\right\}$, here $\varphi$ is the electric potential; $\left\{\mathbf{u}_{p}\right\}$ is the vector of piezoelectric element displacements; $\left\{\mathbf{u}_{s}\right\}$ is the generalized displacement vector of the plate, which includes rotation angles; $\rho$ is the material density; $\left\{\mathbf{t}_{s}\right\}$ is the surface traction vector caused by the interaction with fluid on the part of the plate surface $S_{\sigma}$.

The bottom electrode-covered surface of the piezoelectric element $S_{b o t}$ is grounded and has a zero-value electric potential. The corresponding boundary condition can be written as $\varphi=0$. On the electrode-free parts of the surface of piezoelectric body there are no free electric charges. When an external voltage supply is absent, the other parts of the electrode-covered surfaces of the peizoelement are considered free. In this case, the open circuit mode (oc) is realized. On the other hand, it can be considered as a zero-value electric potential, in which case the short- circuit mode is realized (sc).

The small amplitude vibrations of an ideal compressible fluid in the region $V_{f}$ is described by the well-known Euler equations, continuity equation and equation of state. The elimination of velocity from these equations leads to the Helmholtz equation governing the hydrodynamic pressure $p$, which together with the boundary conditions and the impermeability condition are converted into a weak form using the Bubnov - Galerkin method [18]. Finally, we have

$$
\int_{V_{f}} \delta p\left(\frac{1}{c^{2}} \ddot{p}+\nabla^{2} p\right) d V+\rho_{f} \int_{S_{\sigma}} \delta p \ddot{w} d S=0,
$$

where $c$ is the speed of sound in the liquid medium, $w$ is the normal displacement of the plate and $\rho_{f}$ the density of the fluid.

To take into account the fluid-structure interaction, the traction integral in equation (1) can be expressed as

$$
\int_{S_{\sigma}}\left\{\delta \mathbf{u}_{s}\right\}^{\mathrm{T}}\left\{\mathbf{t}_{s}\right\} d S=\int_{S_{\sigma}}\left\{\delta \mathbf{u}_{s}\right\}^{\mathrm{T}}\left\{\mathbf{n}_{f}\right\} p d S,
$$

where $\left\{\mathbf{n}_{f}\right\}$ is the vector of the outward normal to the fluid region.

In the proposed mathematical model, we assume that sloshing of the free surface of the fluid $\left(S_{\text {free }}\right)$ is absent. The corresponding boundary condition is written as $p=0$. At the interface between the fluid and the rigid wall $S_{w}$, the condition $\left\{\mathbf{n}_{f}\right\}^{\mathrm{T}}\{\dot{\mathbf{v}}\}=0$ is fulfilled.

Let us consider a perturbed motion of the fluid and the plate with the attached piezoelectric element defined as

$$
\mathbf{U}(\mathbf{x}, t)=\left\{\mathbf{u}_{p}(\mathbf{x}, t), \varphi(\mathbf{x}, t), \mathbf{u}_{s}(\mathbf{x}, t), p(\mathbf{x}, t)\right\}=\tilde{\mathbf{U}}(\mathbf{x}) \mathrm{e}^{i \lambda t}, \quad \mathbf{x}=\{x, y, z\},
$$

where $\tilde{\mathbf{U}}(\mathbf{x})$ is the function of coordinates, $\lambda=\omega+i \gamma$ the characteristic parameter, $\omega$ corresponds to the circular natural frequency of vibrations and $\gamma$ is the rate of its damping. Taking into account the above mentioned form of solution instead (1) and (2) we have (wavy line is dropped) 


$$
\begin{gathered}
\int_{V_{p}}\left\{\delta \boldsymbol{\varepsilon}_{p}\right\}^{\mathrm{T}}\left[\mathbf{D}_{p}\right]\left\{\boldsymbol{\varepsilon}_{p}\right\}-\left\{\delta \boldsymbol{\varepsilon}_{p}\right\}^{\mathrm{T}}[\mathbf{e}]\{\mathbf{E}\}-\left\{\delta \mathbf{E}^{\mathrm{T}}[\mathbf{e}]^{\mathrm{T}}\left\{\boldsymbol{\varepsilon}_{p}\right\}-\left\{\delta \mathbf{E}^{\mathrm{T}}[\boldsymbol{\beta}]^{\mathrm{T}}\{\mathbf{E}\}\right) d V-\right. \\
-\lambda^{2} \int_{V_{p}}\left\{\delta \mathbf{u}_{p}\right\}^{\mathrm{T}} \rho_{p}\left\{\mathbf{u}_{p}\right\} d V+\int_{S}\left\{\delta \boldsymbol{\varepsilon}_{s}\right\}^{\mathrm{T}}\left[\mathbf{D}_{s}\right]\left\{\boldsymbol{\varepsilon}_{s}\right\} d S-\lambda^{2} \int_{V_{s}}\left\{\delta \mathbf{u}_{s}\right\}^{\mathrm{T}} \rho_{s}\left\{\ddot{\mathbf{u}}_{s}\right\} d V-\int_{S_{\sigma}}\left\{\delta \mathbf{u}_{s}\right\}^{\mathrm{T}} p\left\{\mathbf{n}_{f}\right\} d S=0 \\
\int_{V_{f}} \delta p\left(-\frac{\lambda^{2}}{c^{2}} p+\nabla^{2} p\right) d V-\lambda^{2} \rho_{f} \int_{S_{\sigma}} \delta p w d S=0 .
\end{gathered}
$$

As a criterion for evaluating the effectiveness of using the piezoelectric element for suppression of a single specified mode of structure vibrations, we use the coefficient of electromechanical coupling proposed in [2] and determined by the formula

$$
K=\sqrt{\frac{\omega_{\mathrm{oc}}^{2}-\omega_{\mathrm{sc}}^{2}}{\omega_{\mathrm{sc}}^{2}}},
$$

where $\omega_{\mathrm{oc}}$ and $\omega_{\mathrm{sc}}$ are the natural frequencies of vibrations of the system in open circuit and short circuit modes corresponding to a given vibration mode. The maximum value of the coefficient $K$ determines the best of possible locations of the piezoelectric element.

In this paper, the coordinates of the location of the piezoelectric element, satisfying the specified constraints and providing the maximum value of the parameter $K$, are found by solving the problem of determining the maximum of the objective function

$$
F\left(\mathbf{x}_{p}\right)=K,
$$

where $\left\{\mathbf{x}_{p}\right\}=\left\{x_{p}, y_{p}\right\}$ are the coordinates of the prescribed point of the piezoelectric element, uniquely determining its location on the plate surface. The restrictions imposed on the parameter $\left\{\mathbf{x}_{p}\right\}$ can take the form of equalities and inequalities that specify the admissible region of variation.

\section{NUMERICAL FORMULATION}

The application of the finite element method $[15,18,19]$ to equations $(5),(6)$ leads to generalized eigenvalue problem, which is written in the matrix form as

$$
\left([\mathbf{K}]-\lambda^{2}[\mathbf{M}]\right)\{\tilde{\mathbf{U}}\}=0,
$$

where the stiffness matrix $[\mathbf{K}]$ and the mass matrix $[\mathbf{M}]$ are given as

$$
[\mathbf{K}]=\left[\begin{array}{cccc}
{\left[\mathbf{K}_{p}\right]} & {\left[\mathbf{K}_{p \varphi}\right]} & 0 & 0 \\
{\left[\mathbf{K}_{p \varphi}\right]^{\mathrm{T}}} & {\left[\mathbf{K}_{\varphi}\right]} & 0 & 0 \\
0 & 0 & {\left[\mathbf{K}_{s}\right]} & -[\mathbf{Q}] \\
0 & 0 & 0 & {\left[\mathbf{K}_{f}\right]}
\end{array}\right], \quad[\mathbf{M}]=\left[\begin{array}{cccc}
{\left[\mathbf{M}_{p}\right]} & 0 & 0 & 0 \\
0 & 0 & 0 & 0 \\
0 & 0 & {\left[\mathbf{M}_{s}\right]} & 0 \\
0 & 0 & \rho_{f}[\mathbf{Q}]^{\mathrm{T}} & {\left[\mathbf{M}_{f}\right]}
\end{array}\right] .
$$

Here, the subscript " $f$ " refers to the corresponding variables of the fluid, [Q] is the fluidstructure interaction matrix [18], and all zeroes are the matrices, too. Discretization of the fluid, electroelastic body and plate computational domains was carried out using the spatial 20-node brick and 8-node plane rectangular finite elements with quadratic approximation of nodal unknowns. The neodymium magnet was modeled as a mass element, which is defined at a single node with the specified concentrated mass and rotational inertia.

Since the eigenvalues of the system of equations (9) are purely real, $\lambda=\omega$. They are calculated using the algorithm, which is based on the implicitly restarted Arnoldi method involving the sparse matrix technology for basic operations. 
A numerical solution of the optimization problem (8) was carried out in several ways. In the simplest case, when the position of the piezoelectric element was determined only by a single coordinate $\left(\left\{\mathbf{x}_{p}\right\}=\left\{x_{p}\right\}\right)$, the golden section search algorithm was used. In all other cases we employed the first order optimization method, which is based on the computation of the derivative of the objective function, and the transformation of the constrained optimization problem to the unconstrained one.

\section{RESULTS AND DISCUSSIONS}

In this section, we present the results of determining the optimal location of the piezoelectric element, which provides the best damping of a given vibration mode of the plate of length $L_{S}$, width $W_{s}$, and thickness $h_{s}$, interacting with a fluid. The computational schemes considered in this work are presented in Figure 1. In order to apply the obtained results to further studies, the numerical simulation was performed using the geometry and characteristics of the real experimental setup (see Table 1). For the boundary conditions (BC) we used the following notation: $\mathrm{F}$ is a free edge, $\mathrm{C}$ is a rigidly clamped edge $\left(\left\{\mathbf{u}_{s}\right\}=\{\boldsymbol{0}\}\right)$. The liquid volume was a rectangular prism $200 \mathrm{~mm}$ long, $150 \mathrm{~mm}$ wide and $195 \mathrm{~mm}$ high. In view of the specific features of the excitation device, a light neodymium magnet weighting $0.10 \mathrm{~g}$ was glued to the plate surface at a distance of $x_{\text {mag }}$ from the clamped edge of the plate and $3 \mathrm{~mm}$ away from its long side. In order to reduce the risk of damage of the piezoelectric element during its attachment to the plate, it was located at a distance of $2 \mathrm{~mm}$ from the clamped end of the plate. Thus, a possible location of the piezoelectric element was restricted to a certain region on the free surface of the plate, which led to the following conditions for the variable parameter:

$$
\begin{gathered}
2 \leq x_{p} \leq x_{\text {mag }}-L_{p}-R_{\text {mag }}, \quad \text { for Case 1, } 2 \\
2 \leq x_{p} \leq L_{s}-L_{p}-2, \quad 0 \leq y_{p} \leq W_{s}-W_{p}, \quad\left\{x_{p}, y_{p}\right\} \in \Psi, \quad \text { for Case 3, }
\end{gathered}
$$

where $L_{p}, W_{p}$ are the length and the width of the piezoelectric element, $R_{\text {mag }}$ is the magnet radius, and $\Psi$ is a set, which prevents the piezoelectric element from falling into a neighborhood of the magnet $\Delta=3 \mathrm{~mm}$ (see the light grey square in Figure 1, Case 3).

Case 1

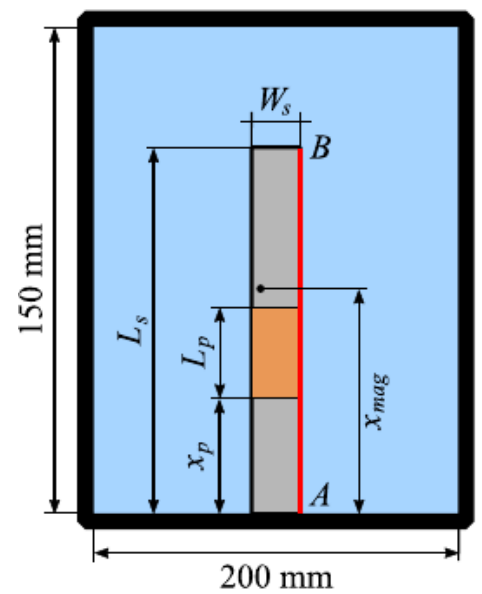

Case 2

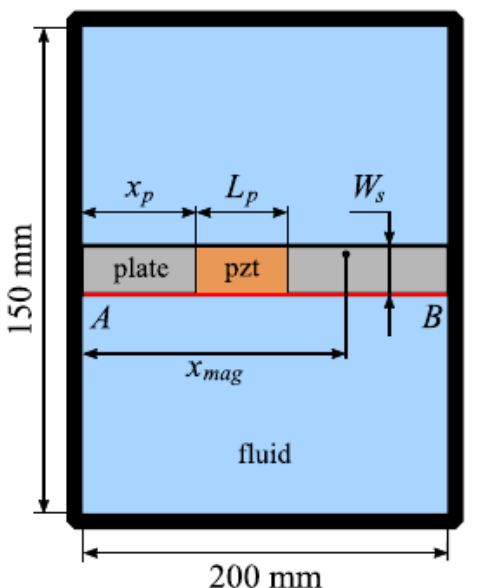

Case 3

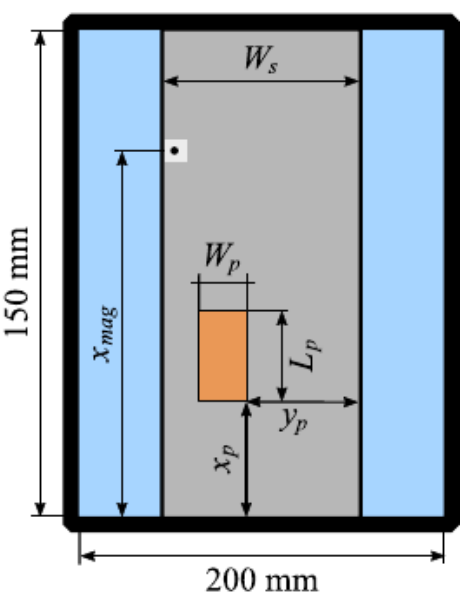

Figure 1. The computational schemes of numerical simulation (top view)

The numerical simulation was made using the following characteristics: for the plate made of duraluminium alloy, the Youngs modulus $E=68.5 \mathrm{GPa}$, Poisson's ratio $v=0.3$, and 
$\rho_{s}=2714 \mathrm{~kg} / \mathrm{m}^{3}$; for the liquid (water) $c=1500 \mathrm{~m} / \mathrm{s}, \rho_{f}=1000 \mathrm{~kg} / \mathrm{m}^{3}$; piezoelectric element was made of PZT-19 piezo-ceramics [20].

Table 1. Geometrical characteristics of plates

\begin{tabular}{|c|c|c|c|c|c|}
\hline Case & $L_{s}, \mathrm{~mm}$ & $W_{s}, \mathrm{~mm}$ & $h_{s}, \mathrm{~mm}$ & $x_{\text {mag }}, \mathrm{mm}$ & $\mathrm{BC}$ \\
\hline 1 & 150 & 20 & 0.94 & 66 & $\mathrm{CF}$ \\
\hline 2 & 150 & 20 & 0.94 & 120 & $\mathrm{CC}$ \\
\hline 3 & 200 & 100 & 0.94 & 130 & $\mathrm{CC}$ \\
\hline
\end{tabular}

\subsection{Plates in air environment}

At the first stage of the study, we determined the natural frequencies and the vibration modes of the plates in air environment. Some results are presented in Table 2, in which the column caption "sc PZT" correspond to the structure with an optimally located piezoelement in the short circuit mode. The classification of the vibration modes $(\mathrm{B}-$ bending, $\mathrm{T}$ - torsional, $\mathrm{M}$ - membrane) and the number of nodal lines in the transverse and longitudinal directions are given in the column headed "Mode". Note that for Case 1 the frequency $\omega_{5}$ corresponds to the plate vibrations in the plane (membrane mode), so that it cannot be damped with the help of a piezoelectric element polarized through thickness. An effective suppression of the torsional vibrations of the examined sample is also impossible, because the electromechanical coupling coefficient $K$ for this mode is very low (Case 1, $\omega_{3}$ ).

Table 2. Natural frequencies of plates in air environment $(\mathrm{Hz})$

\begin{tabular}{|c|c|c|c|c|c|c|c|c|c|}
\hline \multirow{2}{*}{ No. $\omega$} & \multicolumn{3}{|c|}{ Case 1, CF } & \multicolumn{3}{c|}{ Case 2, CC } & \multicolumn{3}{c|}{ Case 3, CC } \\
\cline { 2 - 10 } & Mode & sc PZT & $K$ & Mode & sc PZT & $K$ & Mode & sc PZT & $K$ \\
\hline$\omega_{1}$ & B (0-0) & 41.28 & 0.126 & B (0-0) & 238.98 & 0.105 & B (0-0) & 127.98 & 0.073 \\
\hline$\omega_{2}$ & B (1-0) & 230.34 & 0.101 & B (1-0) & 587.49 & 0.100 & B (0-1) & 206.62 & 0.051 \\
\hline$\omega_{3}$ & T (0-1) & 554.01 & 0.008 & T (0-1) & 1114.47 & 0.046 & B (1-0) & 342.18 & 0.061 \\
\hline$\omega_{4}$ & B (2-0) & 588.58 & 0.097 & B (2-0) & 1212.53 & 0.108 & B (1-1) & 451.33 & 0.052 \\
\hline$\omega_{5}$ & $\mathrm{M}(0-0)$ & - & - & B (3-0) & 2008.56 & 0.105 & B (0-2) & 618.53 & 0.102 \\
\hline
\end{tabular}

A piezoelectric element located on the surface of the plate affects its dynamic characteristics due to the added mass and local increase in stiffness. In the first case, a decrease in the natural frequencies of vibrations is observed, and in the second case, there is an increase of the frequency. Which of these two effects prevails is the matter of particular structure configuration and kinematic boundary conditions. This can be illustrated by the example of a cantilevered plate (see Table 2, Case 1 and Figure 2,a). The location of the piezoelectric element near its clamped end significantly increases the rigidity of the system, which results in an increase of the frequencies $\omega_{1}-\omega_{3}$. As the piezoelectric element is moved closer to the free end of the plate, the effect of the added mass becomes dominant, which leads to a decrease in the frequency of $\omega_{4}$. In the case of a plate clamped at both ends (Case $2)$, the frequency spectrum $\omega_{1}-\omega_{5}$ increases.

Figures 2, 3 compare the vibration modes of the plate with and without a piezoelectric element (indicated by a thick solid line). The normalized displacements $\bar{w}_{i}=w_{i} / \max \left(w_{i}\right)$ shown in Figure 2 were the displacements obtained along the straight line AB (Figure 1). The location of the magnet is denoted by a vertical dotted line in Figure 2 and by circle in 
Figure 3. The results suggest that the bending vibration modes do not show qualitative changes. Figure 2 shows that due to the attachment of the piezoelectric element, the amplitude of the deflection decreases, and the displacement of nodal lines takes place. The most significant changes up to a full transformation are observed in the torsional modes. An example is the mixed vibration mode for the plate clamped at both ends, for which the number of half-waves along each side in the direction of the Ox-axis is different (Figure 3, Case 2, $\left.\omega_{3}\right)$.
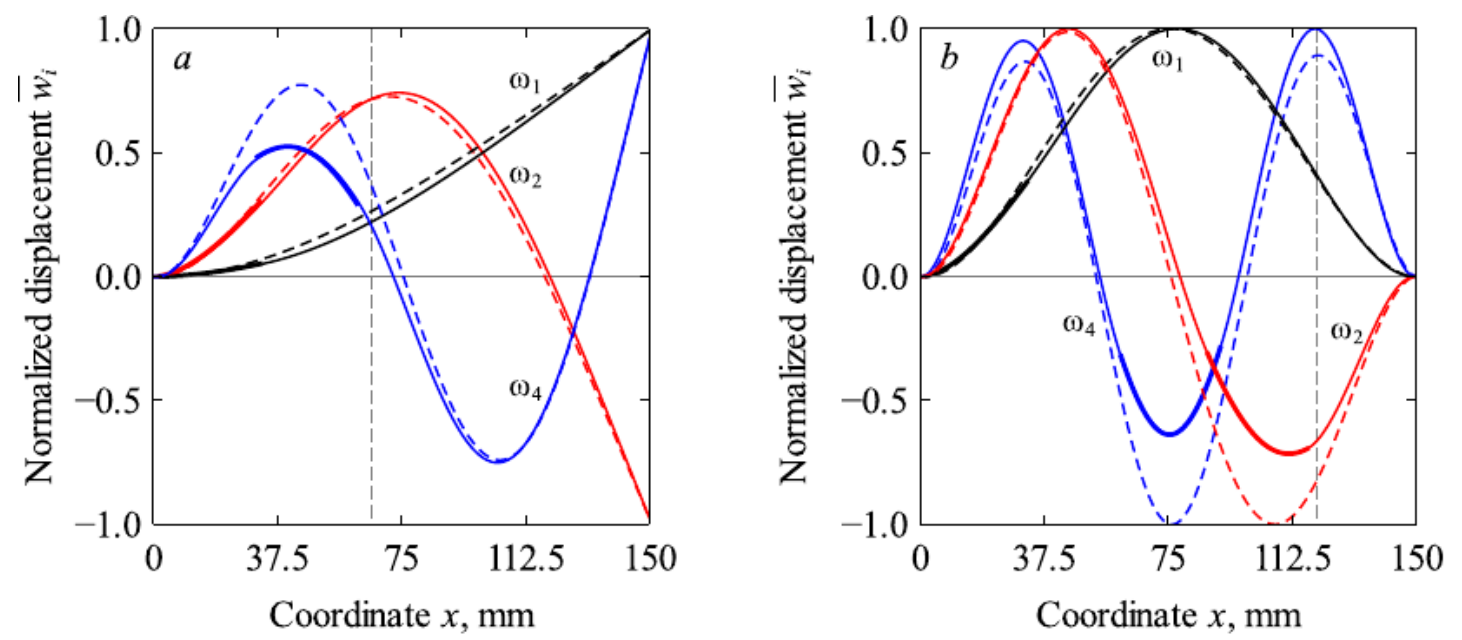

Figure 2. Normalized modes of vibration of the cantilever $(a)$ and clamped-clamped $(b)$ plates with (solid lines) and without piezoelectric element (dashed lines)

Table 3 shows the values of $\mathbf{x}_{p}=\left\{x_{p}, y_{p}\right\}$, which determine the optimal location of the piezoelectric element, providing maximum damping of the vibration mode $\omega_{i}$.

Case 1, CF

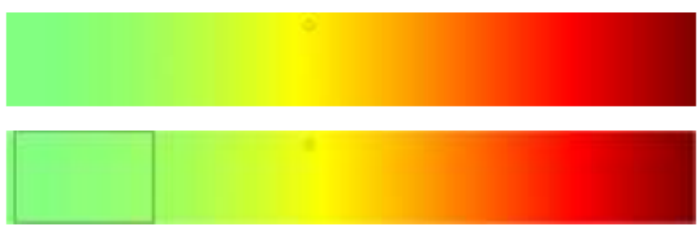

$\omega_{1}: 33.78 \mathrm{~Hz} / 41.28 \mathrm{~Hz}$

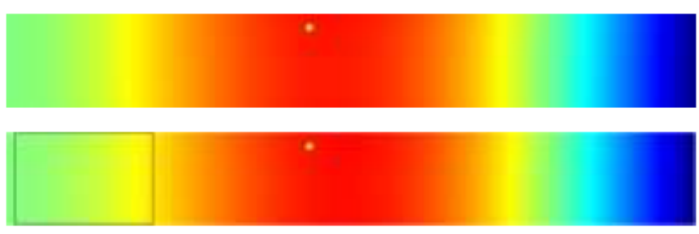

$\omega_{2}: 209.24 \mathrm{~Hz} / 230.34 \mathrm{~Hz}$

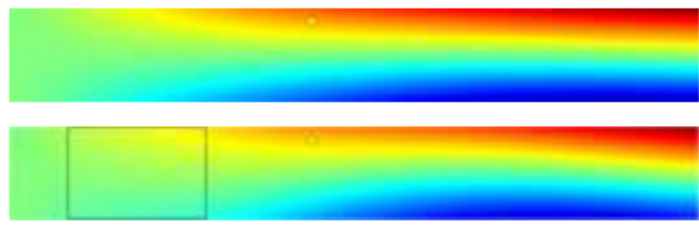

$\omega_{3}: 488.23 \mathrm{~Hz} / 554.01 \mathrm{~Hz}$
Case 2, CC

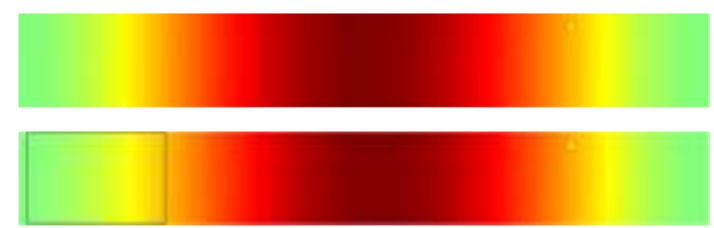

$\omega_{1}: 212.76 \mathrm{~Hz} / 238.98 \mathrm{~Hz}$

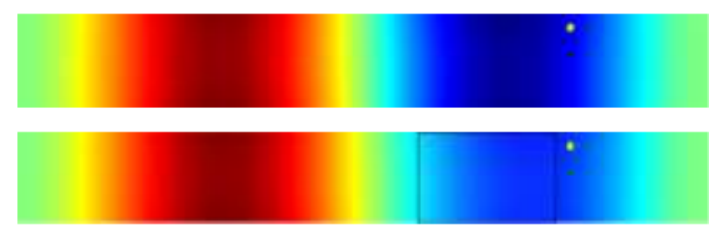

$\omega_{2}: 582.07 \mathrm{~Hz} / 587.49 \mathrm{~Hz}$

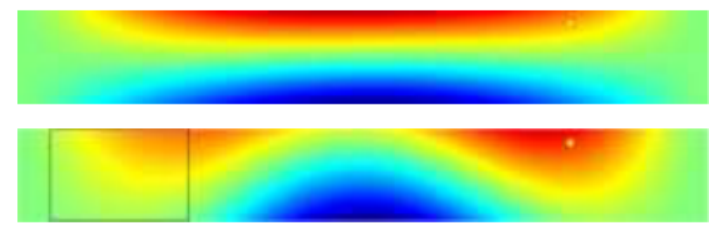

$\omega_{3} 999.06 \mathrm{~Hz} / 1114.47 \mathrm{~Hz}$ 


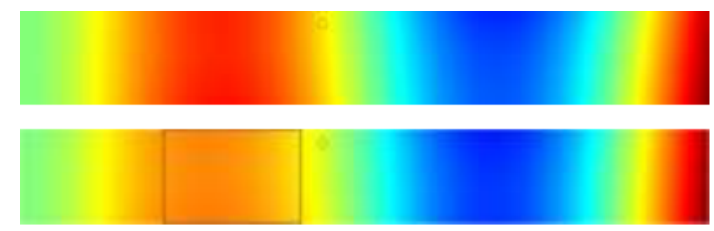

$\omega_{4}: 592.19 \mathrm{~Hz} / 588.58 \mathrm{~Hz}$

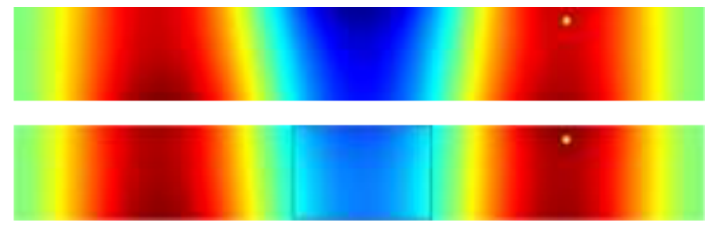

$\omega_{4}: 1136.12 \mathrm{~Hz} / 1212.53 \mathrm{~Hz}$

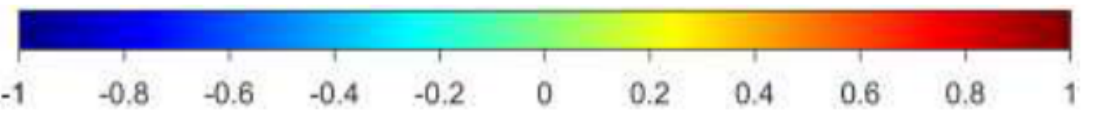

Figure 3. Comparison of the vibration modes of the plates with and without piezoelectric element. On a spectral scale, colors denote the normalized displacement $\bar{w}_{i}$

Table 3. Optimal positions $\mathbf{x}_{p}$ of piezoelement on plates in air environment ( $\left.\mathrm{mm}\right)$

\begin{tabular}{|c|c|c|c|c|c|c|}
\hline \multirow{2}{*}{ No. $\omega$} & \multicolumn{2}{|c|}{ Case $1, \mathrm{CF}$} & \multicolumn{2}{c|}{ Case 2, CC } & \multicolumn{2}{c|}{ Case 3, CC } \\
\cline { 2 - 7 } & Mode & $\mathrm{x}_{p}$ & Mode & $\mathrm{x}_{p}$ & Mode & $\mathrm{x}_{p}$ \\
\hline$\omega_{1}$ & $\mathrm{~B}(0-0)$ & $\{2.0,0.0\}$ & $\mathrm{B}(0-0)$ & $\{2.0,0.0\}$ & $\mathrm{B}(0-0)$ & $\{168.0,43.0\}$ \\
\hline$\omega_{2}$ & $\mathrm{~B}(1-0)$ & $\{2.0,0.0\}$ & $\mathrm{B}(1-0)$ & $\{87.0,0.0\}$ & $\mathrm{B}(0-1)$ & $\{2.0,0.9\}$ \\
\hline$\omega_{3}$ & $\mathrm{~T}(0-1)$ & $\{12.8,0.0\}$ & $\mathrm{T}(0-1)$ & $\{7.1,0.0\}$ & $\mathrm{B}(1-0)$ & $\{47.0,47.5\}$ \\
\hline$\omega_{4}$ & $\mathrm{~B}(2-0)$ & $\{31.1,0.0\}$ & $\mathrm{B}(2-0)$ & $\{60.7,0.0\}$ & $\mathrm{B}(1-1)$ & $\{43.3,3.9\}$ \\
\hline
\end{tabular}

\subsection{Plates located on the free surface of the fluid}

When the plate interacts with the fluid layer from bottom, a decrease in the natural frequencies of vibrations, especially in the lowest ones can reach and even exceed $50 \%$ (compare Table 2 and Table 4). At the same time, there is no qualitative change in the modes of vibrations, but their order in the spectrum is disturbed, and there is a small shift in the nodal lines. In this regard, it is advisable to evaluate the effect of hydroelastic interaction on the optimal location of the piezoelectric element on the surface of a plate located on the fluid layer.

Table 4. Natural frequencies of plates located on the free surface of the fluid $(\mathrm{Hz})$

\begin{tabular}{|c|c|c|c|c|c|c|c|r|c|}
\hline \multirow{2}{*}{ No. $\omega$} & \multicolumn{3}{|c|}{ Case 1, CF } & \multicolumn{3}{c|}{ Case 2, CC } & \multicolumn{3}{c|}{ Case 3, CC } \\
\cline { 2 - 10 } & Mode & sc PZT & $K$ & Mode & sc PZT & $K$ & Mode & sc PZT & $K$ \\
\hline$\omega_{1}$ & B (0-0) & 22.46 & 0.126 & B $(0-0)$ & 125.97 & 0.105 & B $(0-0)$ & 31.68 & 0.073 \\
\hline$\omega_{2}$ & B (1-0) & 128.23 & 0.100 & B $(1-0)$ & 342.24 & 0.099 & B (0-1) & 82.55 & 0.048 \\
\hline$\omega_{3}$ & B (2-0) & 355.62 & 0.096 & B (2-0) & 703.16 & 0.107 & B (1-0) & 108.96 & 0.060 \\
\hline$\omega_{4}$ & $\mathrm{~T}(0-1)$ & 395.41 & 0.002 & T $(0-1)$ & 686.73 & 0.064 & B (1-1) & 194.03 & 0.052 \\
\hline
\end{tabular}

As an example, consider the computational schemes shown in Figure 1. A comparison between the results presented in Table 3 and Table 5 demonstrates that in Cases 1, 2 the optimal values of the parameter $\mathbf{x}_{p}$ for the plate in air environment and the plate located on the fluid layer either coincide or differ in the limits of relative error equal to $2 \%$. In this case, the coefficients of the electromechanical coupling $K$ also differ by no more than $2 \%$. This can occur only in the absence of significant qualitative changes in the vibration modes and at the location of the piezoelement far from the nodal lines. The case of the torsional mode should be considered separately. It has been found that for a cantilevered plate (Case 1) this mode cannot be effectively damped, because $K$ is too small, whereas for a plate clamped at two ends 
(Case 2), this proves to be possible ( $K=0.046$ in air and $K=0.064$ on the layer of fluid). In the third case (Case 3 ), the coordinates of the location of the piezoelectric element differ more significantly, but the change in the coefficient of electromechanical coupling is still no more than $2 \%$. The exception is the mode B (0-1), which falls under the effect of the fluid near the plate edge, so that the value of $K$ is somewhat lower. Note that the accuracy of gluing the piezoelectric element by hand is often $\pm 0.5 \mathrm{~mm}$, which implies that value of tenths of a millimeter obtained in the numerical calculations is simply unfeasible.

Table 5. Optimal positions $\mathbf{x}_{p}$ of piezoelement on plates located on the fluid layer (mm)

\begin{tabular}{|c|c|c|c|c|c|c|}
\hline \multirow{2}{*}{ No. $\omega$} & \multicolumn{2}{|c|}{ Case 1, CF } & \multicolumn{2}{c|}{ Case 2, CC } & \multicolumn{2}{c|}{ Case 3, CC } \\
\cline { 2 - 7 } & Mode & $\mathrm{x}_{p}$ & Mode & $\mathrm{x}_{p}$ & Mode & $\mathrm{x}_{p}$ \\
\hline$\omega_{1}$ & $\mathrm{~B}(0-0)$ & $\{2.0,0.0\}$ & $\mathrm{B}(0-0)$ & $\{2.0,0.0\}$ & $\mathrm{B}(0-0)$ & $\{168.0,39.7\}$ \\
\hline$\omega_{2}$ & $\mathrm{~B}(1-0)$ & $\{2.0,0.0\}$ & $\mathrm{B}(1-0)$ & $\{87.0,0.0\}$ & $\mathrm{B}(0-1)$ & $\{2.0,0.7\}$ \\
\hline$\omega_{3}$ & $\mathrm{~B}(2-0)$ & $\{31.5,0.0\}$ & $\mathrm{B}(2-0)$ & $\{60.2,0.0\}$ & $\mathrm{B}(1-0)$ & $\{45.7,40.9\}$ \\
\hline$\omega_{4}$ & $\mathrm{~T}(0-1)$ & $\{29.9,0.0\}$ & $\mathrm{T}(0-1)$ & $\{52.9,0.0\}$ & $\mathrm{B}(1-1)$ & $\{45.9,4.7\}$ \\
\hline
\end{tabular}

\section{CONCLUSION}

A series of numerical calculations conducted during this study allowed us to identify situations where the hydroelastic interaction can be ignored in the search for the optimal position of the piezoelectric element on the surface of the plate, which is located on the fluid layer. Such assumption introduces a minor relative error (about $2 \%$ ), and significantly reduces the computation time. In the proposed mathematical model, the effect of the fluid can be neglected, for example, in the case when the mode shape of the plate along the direction of one of the axes does not significantly differ from that of the beam. In other cases, the degree of the fluid effect on the value of the optimal parameter is determined by the specific features of the vibration mode being considered and depends on a number of factors (geometrical dimensions, boundary conditions, etc.). Nowadays, piezoelectric elements attached to the surface of various structures are generally used for passive and active suppression of hydroelastic vibrations. Therefore, consideration of the fluid effect is a necessity dictated by the fact it significantly reduces the spectrum of natural frequencies.

The study was supported by the grant of the Russian Scientific Foundation (project No. 18-71-10054).

\section{REFERENCES}

[1] Forward, R. L. Electronic damping of vibrations in optical structures. Applied Optics, 18, 1979, pp.690-697.

[2] Hagood, N. W., Von Flotow, A. Damping of structural vibrations with piezoelectric materials and passive electrical networks. Journal of Sound and Vibration, 146(2), 1991, pp. 243-268.

[3] Belloli, A., Ermanni, P. Optimum placement of piezoelectric ceramic modules for vibration suppression of highly constrained structures. Smart Materials and Structures, 16(5), 2007, pp.1662-1671.

[4] Flynn, E. B., Todd, M. D. Optimal placement of piezoelectric actuators and sensors for detecting damage in plate structures. Journal of Intelligent Material Systems and Structures, 21, 2010, pp. 265-273.

[5] Lee, S., Youn, B. D. A design and experimental verification methodology for an energy harvester skin structure. Smart Materials and Structures, 20, 2011, 057001. 
[6] Ramesh, K., Narayanan S. The optimal location of piezoelectric actuators and sensors for vibration control of plates. Smart Materials and Structures, 16, 2007, pp. 2680-2691.

[7] Fein, O. M. A model of piezo-resitive damping of two-dimensional structures, Journal Sound and Vibration, 310, 2008, pp. 865-880.

[8] Bachmann, F., Bergamini, A., Ermanni, P. Optimal piezoelectric positioning a strain-energy based finite element approach, Journal of Intelligent Material Systems and Structures, 23(14), 2012, pp. $1575-1591$.

[9] Lin, Y. J., Venna, S. V. A novel method for piezoelectric transducers placement for passive vibration control of geometrically non-linear structures, Sensor Review, 28, 2008, pp. 233-241.

[10] Sevodina, N. V., Yurlova, N. A., Oshmarin, D. A. The optimal placement of the piezoelectric element in a structure based on the solution of the problem of natural vibrations. Solid State Phenomena, 243, 2015, pp. 67-74.

[11] Hollkamp, J. J. Multimodal passive vibration suppression with piezoelectric materials and resonant shunts, Journal of Intelligent Material Systems and Structures, 5, 1994, pp. 49-57.

[12] Trindade, M. A., Benjeddou, A. Effective electromechanical coupling coefficients of piezoelectric adaptive structures: critical evaluation and optimization, Mechanics of Advanced Materials and Structures, 16(3), 2009, pp. 210-223.

[13] Ducarne, J., Thomas, O., Deu, J.-F. Placement and dimension optimization of shunted piezoelectric patches for vibration reduction, Journal Sound and Vibration, 331, 2013, pp. 3286-3303.

[14] Matveenko, V. P., Iurlova, N. A., Oshmarin, D. A., Sevodina, N. V. and Iurlov, M. A. An approach to determination of shunt circuits parameters for damping vibrations. International Journal of Smart and Nano Materials, 9(2), 2018, pp. 135-149.

[15] Reddy, J. N. An introduction to nonlinear finite element analysis, 2nd edition. Oxford: Oxford University Press, 2015, pp. xvii+463.

[16] Washizu, K. Variational methods in elasticity and plasticity. London: Pergamon Press, 1982, pp. 630 .

[17] IEEE Standard on Piezoelectricity. ANSI/IEEE Std 176-1987, 1988.

[18] Zienkiewicz, O. C. and Taylor, R. L. The finite element method, vols. $1-2,5^{\text {th }}$ edition. Oxford: Butterworth-Heinemann, 2000.

[19] Allik, H., Hughes, J. R. Finite element for piezoelectric vibration. International Journal Numerical Methods of Engineering, 2(2), 1970, pp. 151-157.

[20] Iurlova, N., Sevodina, N., Oshmarin, D., Iurlov, M. Algorithm for solving problems related to the natural vibrations of electro-viscoelastic structures with shunt circuits using ANSYS data. International Journal of Smart and Nano Materials, 10(2), 2019, pp. 156-176.

[21] Tharun K. Boya and Ashok K. Bagha Choice of Optimal Location of Piezoelectric Sensor on Steel Plate Using Mode Shapes. International Journal of Mechanical Engineering and Technology, 8(7), 2017, pp. 1225-1230.

[22] Dr. Hatem Hadi Obeid, Converting Forced Vibrations Induced in Piezoelectric Cantilever Plate into Nano Electrical Power, International Journal of Mechanical Engineering and Technology, 9(11), 2018, pp. 146-160.

[23] R. Ganesh, K. Karthik, A. Manimaran and M. Saleem. Vibration Damping Characteristics of Cantilever Beam Using Piezoelectric Actuator. International Journal of Mechanical Engineering and Technology, 8(6), 2017, pp. 212-221.

[24] D. Rajesh, V. Balaji, A. De varaj and D. Yogaraj, An Investigation on Effects of Fatigue Load on Vibration Characteristics of Woven Fabric Glass/Carbon Hybrid Composite Beam under FixedFree End Condition using Finite Element Method. International Journal of Mechanical Engineering and Technology 8(7), 2017, pp. 85-91. 